\title{
A public participatory GIS and multi criteria decision analysis framework for the evaluation of transport scenarios*
}

\author{
Nadia Giuffrida ${ }^{1 \dagger}$ \\ ${ }^{1}$ Department of Civil Engineering and Architecture, University of Catania, Italy
}

\section{Summary}

The decision-making process of transport projects is very complex, especially for public administrations which have to take into account often incomparable criteria of judgment. In addition, in order to achieve a good social sharing and robustness of the decision, policy makers have to include in the process not only the transport planning experts but also the stakeholders of the community. The purpose of this study is to propose an evaluation framework to support the decision making process, able to allow public participation in the assessment of transport planning and design scenarios while, at the same time, ensuring a high level of technical quality of the final decision. The designed framework includes a method to analyze monetary and non-monetary parameters, easily understandable for all decision makers. It is based on the application of Multi Criteria Decision Analysis (MCDA) techniques, an evaluation process that can take into account different quantitative and qualitative objectives and criteria; in order to favor public participation, the implementation of this technique takes advantage of Geographic Information System (GIS) for its ability to easily represent the impact of spatially based alternative project scenarios. The analysis of several case studies on transport planning and design scenarios allowed to assess the level of implementation of the three main ingredients of the framework: MCDA, GIS and public participation.

Keywords: Transport planning, transport decision-making, public participation.

*Abstract of one of the Best PhD Theses Award, 2020.

${ }^{\dagger}$ E-mail: nadia giuffrida@unict.it 


\section{Riassunto \\ Un Sistema di Informazione Geografica a partecipazione pubblica ed uno schema d'analisi multicriteriale delle decisioni per la valutazione di scenari di trasporto*}

Il processo decisionale dei progetti di trasporto è molto complesso, soprattutto per le pubbliche amministrazioni che devono tener conto di criteri di giudizio spesso incompatibili. Inoltre, al fine di ottenere una buona condivisione sociale e solidità della decisione, chi stabilisce le norme deve includere nel processo non solo gli esperti di pianificazione dei trasporti ma anche gli interessati della comunità. Lo scopo di questo studio è quello di proporre un quadro di valutazione a supporto del processo decisionale, in grado di consentire la partecipazione pubblica alla valutazione degli scenari di pianificazione e progettazione dei trasporti garantendo, allo stesso tempo, un alto livello di qualità tecnica della decisione finale. Lo schema progettato include un metodo per analizzare i parametri monetari e non monetari, facilmente comprensibile per tutti i decisori. $\mathrm{Si}$ basa sull'applicazione di tecniche di analisi multicriteriale della decisione (Multi Criteria Decision Analysis, MCDA), un processo di valutazione che può tenere conto di diversi obiettivi e criteri quantitativi e qualitativi; per favorire la partecipazione del pubblico, l'implementazione di questa tecnica si avvale del Sistema di Informazione Geografica (Geographic Information System, GIS) per la sua capacità di rappresentare facilmente l'impatto di scenari progettuali alternativi basati spazialmente. L'analisi di diversi casi studio sulla pianificazione dei trasporti e sugli scenari di progettazione ha permesso di valutare il livello di implementazione dei tre ingredienti principali del framework: MCDA, GIS e partecipazione pubblica.

Parole chiave: Pianificazione dei trasporti, processo decisionale per $i$ trasporti, partecipazione pubblica.

*Riassunto di una delle Migliori Tesi di Dottorato premiate nel 2020. 